\title{
Microwave Attenuation Measurements With Accuracies From 0.0001 to 0.06 Decibel Over a Range of 0.01 to 50 Decibels
}

\author{
G. F. Engen and R. W. Beatty
}

(January 15, 1960)

\begin{abstract}
The application of certain power stabilization and measurement techniques to the problem of attenuation measurement has yielded a measurement system with a stability and resolution of the order of 0.0001 decibel. A practical application for this technique was recently provided in the calibration of a rotary vane type of variable microwave attenuator

In order to take complete advantage of this increased stability it was necessary to apply refined techniques to the evaluation and reduction of mismatch error.

This proved to be by far the most exacting practical application of the cited techniques encountered to date, but the results of this calibration showed excellent agreement with the mathematically predicted values used in marking the attenuator dial.
\end{abstract}

\section{Introduction}

The following is a report on some recent work at the Radio Standards Laboratories of the National Bureau of Standards at Boulder, Colo. It is believed that this work represents perhaps the most accurate measurements of microwave attenuation yet made.

In the measurement of microwave attenuation, the stability or resolution of the associated measuring system places a limit on the accuracy with which small values of attentuation may be measured, because the reduction of other sources of error (such as mismatch) will tend to make the total error approach this value. In practice this limit has been typically of the order of $\pm 0.01 \mathrm{db}$.

The application of certain power stabilization ${ }^{1}$ and measurement ${ }^{2}$ techniques to the problem of attenuation measurement has yielded a measurement system with a nominal hundredfold increase in resolution and stability. A practical application for demonstrating the capabilities of this technique was recently provided in the calibration of a rotary vane type of variable microwave attenuator which is characterized, in part, by a high degree of resolution for small values of attenuation. Thus when a recent application called for this characteristic in a variable attenuator, and when a preliminary study of the mismatch and stability characteristics of an attenuator of this type indicated that such a step was warranted, it proved desirable to experimentally confirm the mathematically predicted values of attenuation which were engraved on the attenuator dial. This proved to be by far the most exacting practical application of the cited techniques encountered to date.

In order to take complete advantage of the improved stability, refined techniques were applied to the evaluation and reduction of the mismatch error. The capability of the system is indicated by the tabulated results of the attenuation calibration, and the estimate of the limits of error is supported by an analytic and experimental treatment.

1 Glenn F. Engen, Amplitude stabilization of a microwave signal source, IRE Trans. on Misrowave Theory \& Tech. MTTT-6, No. 2, 202-206 (Apr. 1958).

2 Glenn F. Engen, A self-balancing direct-current bridge for accurate bolometric power measurements, J. Research NBS 59, No. 2, 101-105 (Aug. 1957) RP2776. 


\section{The Calibration System}

A simplified diagram of the calibration system is shown in figure 1. The attenuator under test is placed between an amplitude stabilized microwave signal source ${ }^{1}$ and a bolometer mount and power meter. The power meter consists of a self-balancing d-c bolometer bridge ${ }^{2}$ with provisions for measuring and recording the d-c bias power required to maintain the bolometer at its operating resistance of $200 \mathrm{ohms}$. The two bolometer mounts $M_{1}$ and $M_{2}$ shown in the temperature stabilized water bath are for power measurement and signal source stabilization, respectively. A reasonable amount of care was exercised to obtain good performance from each item of equipment, with a resultant system performance as shown in figures $2 \mathrm{a}$ and $2 \mathrm{~b}$. Figure $2 \mathrm{a}$ shows the stability and repeatability with the attenuator alternately set at the 0.00 - and $0.01-\mathrm{db}$ positions. It will be noted that the stability and repeatability are better than $0.0001 \mathrm{db}$ (10 microbels). A recording of the long term system stability is given in figure $2 \mathrm{~b}$ where the maximum variation is of the order of \pm 10 microbels.

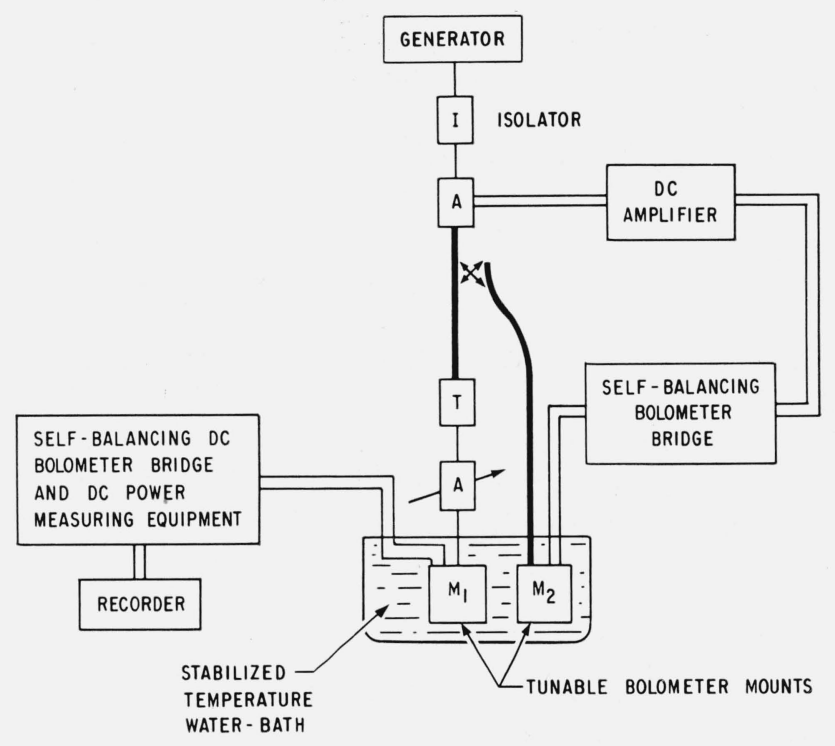

FIGURE 1. Simplified diagram of calibration system.

The absolute accuracy of measurement of small changes in d-c power at this level is estimated to be of the order of $0.02 \mu \mathrm{w}$. This indicates that further improvement could be expected in the results if the system stability could be improved in some manner, perhaps by use of a frequency-stabilized signal source.

\section{Theory of Measurement}

One could make an attenuation measurement by measuring the microwave powers $P_{1}$ and $P_{2}$ absorbed by the bolometer mount $M_{1}$ when the attenuator is set first on zero, then to some other setting. The relative attenuation $A$ at this setting is

$$
A=10 \log _{10} \frac{P_{1}}{P_{2}}
$$




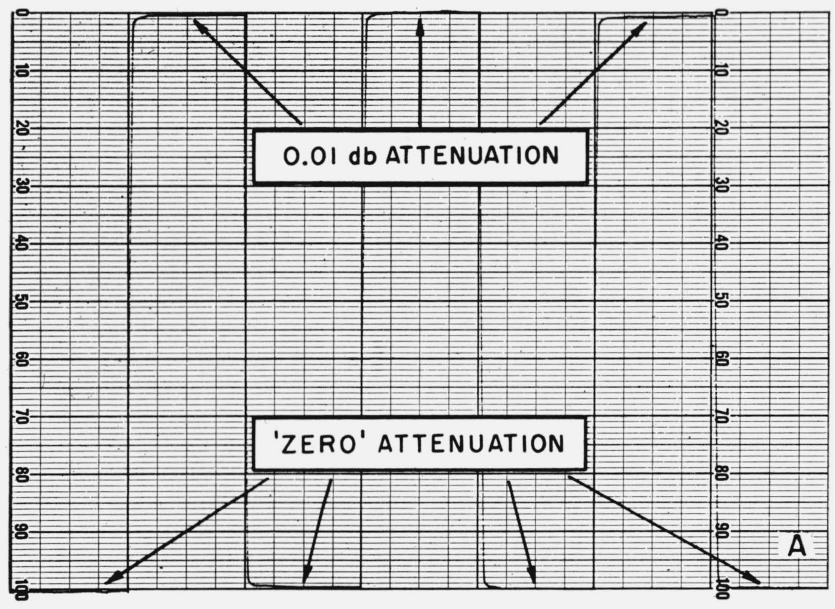

Figure 2a. System response to a $0.01 \mathrm{db}$ change in attenuation.

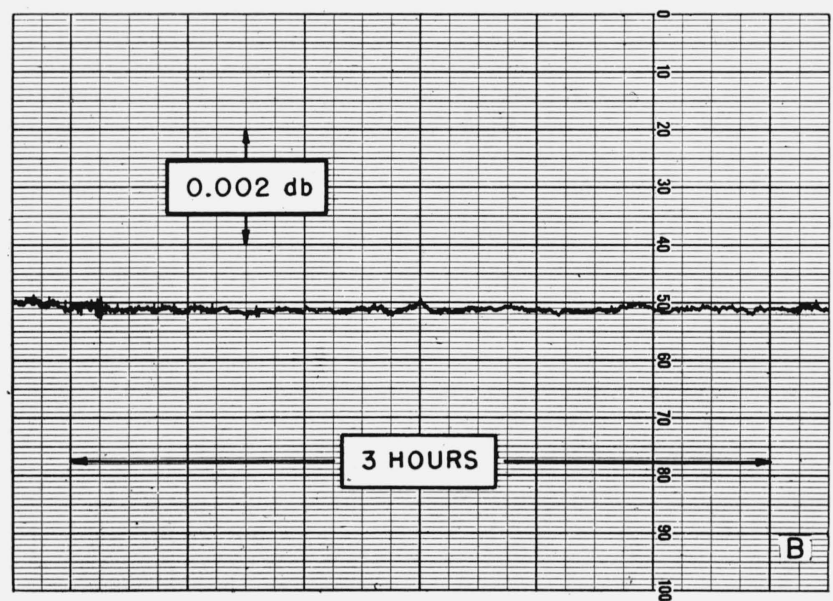

FIGURE 2b. Long-term system stability.

In making such a measurement, one assumes that the microwave power $P$ absorbed by the bolometer element is proportional ${ }^{3}$ to the d-c power $W$ withdrawn in order to keep the bolometer resistance constant. Letting $W_{0}$ represent the $\mathrm{d}$-c power required to bias the bolometer at its operating resistance when $P$ equals zero,

$$
A=10 \log _{10} \frac{W_{0}-W_{1}}{W_{0}-W_{2}}
$$

where $W_{1}$ and $W_{2}$ are the d-c bias powers corresponding to $P_{1}$ and $P_{2}$. (For the bolometer used, $W_{0} \approx 15 \mathrm{mw}$ and $P_{1} \approx 10 \mathrm{mw}$.)

The apparatus employed permitted direct measurements of differences in d-c power, a procedure permitting greater accuracy and convenience than calculation of differences from separate measurements. The changes in d-c power level during an attenuation measurement are shown in figure 3.

${ }^{3}$ The constant of proportionality is determined by the substitution error of the bolometer which is known from other experimental data to be independent of power level. 

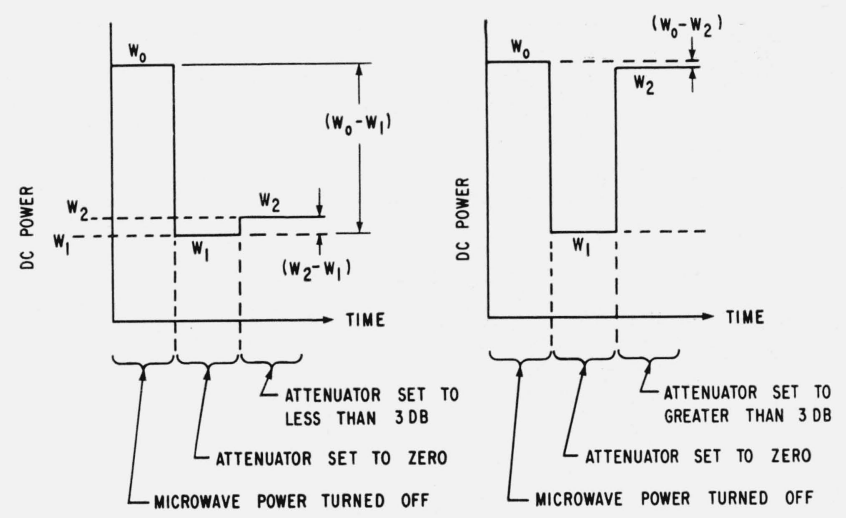

FiguRE 3. Changes in d-c power level during an attenuation measurement.

For attenuations less than approximately $3 \mathrm{db}$, the power difference $W_{2}-W_{1}$ was measured directly. If we let $W_{21}=W_{2}-W_{1}$, and $W_{01}=W_{0}-W_{1}$, eq (2) may be written

$$
A=10 \log _{10}\left(\frac{1}{1-\frac{W_{21}}{W_{01}}}\right) \text {. }
$$

For attenuations greater than approximately $3 \mathrm{db}$, the power difference $W_{0}-W_{2}$ or $W_{02}$ was measured directly. Equation (2) becomes

$$
A=10 \log _{10} \frac{W_{01}}{W_{02}}
$$

\section{Propagation of Error in Measuring D-C Power Differences}

It is estimated that the error in measuring d-c power differences is within 0.1 percent $+0.1 \mu \mathrm{w}$. When $W_{2}-W_{1}$ is measured, it can be shown that the limit of error in determining the attenuation is

$$
\epsilon=10 \log _{10}\left[\frac{1}{1-\frac{1}{1-\frac{W_{21}}{W_{01}}}\left(\frac{0.1 \mu \mathrm{w}}{W_{01}}+0.001 \frac{W_{21}}{W_{01}}\right)}\right]
$$

and when $W_{2}-W_{0}$ is measured,

$$
\epsilon=10 \log _{10}\left[\frac{1}{1-\frac{0.1 \mu \mathrm{w}+0.001 W_{02}}{W_{02}}}\right] .
$$

The calculated limits of error are shown in figure 4.

\section{Mismatch Errors}

The mismatch error ${ }^{4}$ in calibrating a variable attenuator depends upon the reflections from the system in which the attenuator is placed and upon the changes in characteristics of the attenuator as its dial is moved from the reference position. The graph of figure 4 shows calculated

${ }^{4}$ R. W. Beatty, Mismatch errors in the measurement of ultrahigh-frequency and microwave variable attenuators, J. Research NBS 52, No. 1, 7-9 (1954) RP2465. 


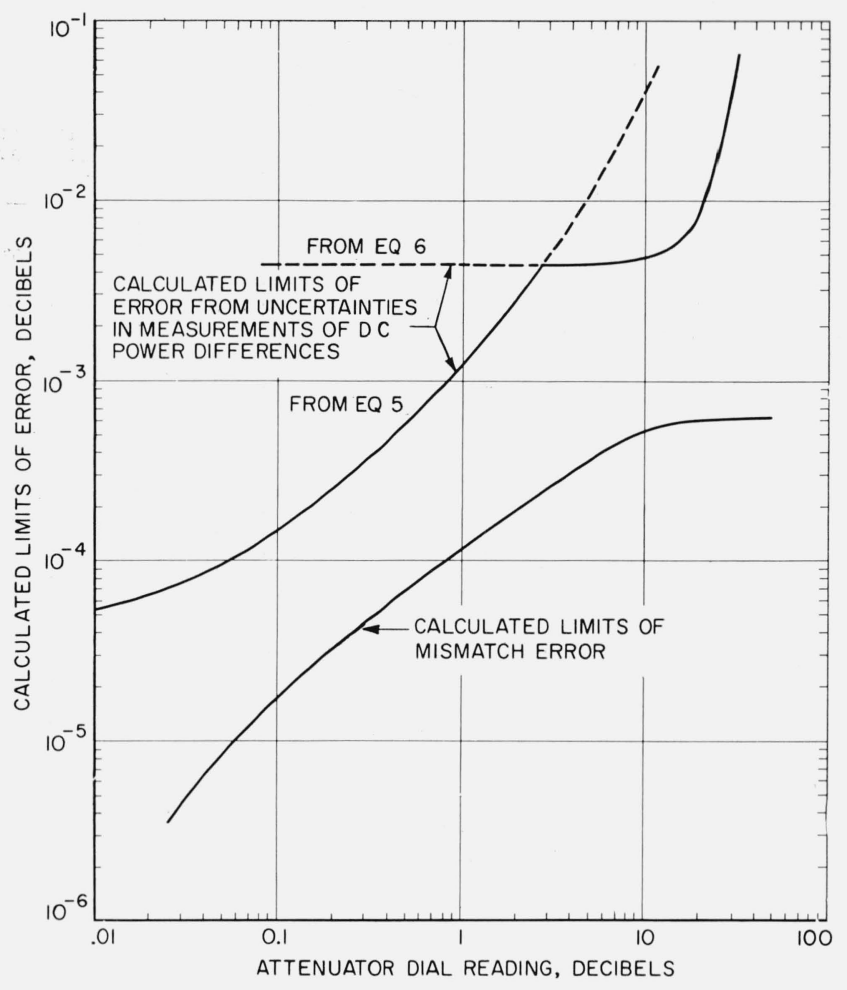

FiguRE 4. Limits of error as a function of attenuation.

limits of error ${ }^{5}$ for the attenuator used, based upon measurements of the magnitude of the changes in the scattering coefficients $S_{11}$ and $S_{22}$ of the attenuator. It was assumed that there was no phase change in $S_{21}$. The mismatch error is below $0.0001 \mathrm{db}$ for attenuator settings up to $0.1 \mathrm{db}$, and remains below $0.001 \mathrm{db}$ for higher settings.

\section{Results}

The calibration data taken at $9.3897 \mathrm{kMc}$ is shown in table 1 . Three sets of data are shown in order to give an idea of the resettability of the attenuator, and more significant figures are given than one can normally use when interpolating between the marked dial divisions. It should be noted that the calibration is not necessarily representative of this type of attenuator, because only one such attenuator was calibrated by this procedure, and this was done at only one frequency. It should also be noted that this type of calibration is not available on a routine basis, because there are as yet no commercially available attenuators which can be read and are repeatable to $0.0001 \mathrm{db}$.

The estimated limit of error for the complete range of the attenuator, as determined from figure 4, is also shown in table 1 . Above $20 \mathrm{db}$, the calibration was made in two parts : Measurement of the $20 \mathrm{db}$ step, and measurement of the additional attenuation referred to this step. For these values, the quoted limit of error is the sum of the errors in the individual steps. The accuracy of setting the attenuator dial on the marks is not as good as the accuracy of the measurements.

It is noted that at the low end, the estimated accuracy of the measurement is also better than the repeatability of setting the attenuator on the mark. One concludes that further improvements in attenuators need to be made if we are to take full advantage of the most accurate measurement techniques.

${ }^{5}$ See appendix. 
TABLE 1. Results of three sets of measurements and calculated errors in single measurements

\begin{tabular}{|c|c|c|c|c|c|c|}
\hline \multirow{2}{*}{$\begin{array}{l}\text { Attenuator } \\
\text { dial } \\
\text { reading }\end{array}$} & \multicolumn{4}{|c|}{ Measured attenuations corresponding to dial reading } & \multirow{2}{*}{$\begin{array}{l}\text { Maximum devia- } \\
\text { tion from average }\end{array}$} & \multirow{2}{*}{$\begin{array}{l}\text { Calculated limit } \\
\text { of error in single } \\
\text { measurements }\end{array}$} \\
\hline & 1 & 2 & 3 & Avg & & \\
\hline $\begin{array}{c}d b \\
0.01 \\
.02 \\
.03 \\
.04 \\
.05\end{array}$ & $\begin{array}{c}d b \\
0.01077 \\
.02123 \\
.03015 \\
.04043 \\
.05181\end{array}$ & $\begin{array}{c}d b \\
0.01083 \\
.02145 \\
.03035 \\
.04088 \\
.05216\end{array}$ & $\begin{array}{c}d b \\
0.01044 \\
.02145 \\
.03038 \\
.04074 \\
.05226\end{array}$ & $\begin{array}{c}d b \\
0.0107 \\
.0214 \\
.0303 \\
.0407 \\
.0521\end{array}$ & $\begin{array}{c}d b \\
0.0003 \\
.0002 \\
.0001 \\
.0003 \\
.0003\end{array}$ & $\begin{array}{c}d b \\
0.000055 \\
.000068 \\
.000080 \\
.000091 \\
.00010\end{array}$ \\
\hline $\begin{array}{l}.06 \\
.07 \\
.08 \\
.09 \\
.1\end{array}$ & $\begin{array}{l}.06102 \\
.06992 \\
.07991 \\
.09075 \\
.10226\end{array}$ & $\begin{array}{l}.06067 \\
.07027 \\
.08056 \\
.09104 \\
.10203\end{array}$ & $\begin{array}{l}.06086 \\
.06985 \\
.08017 \\
.09078 \\
.10210\end{array}$ & $\begin{array}{l}.0609 \\
.0700 \\
.0802 \\
.0909 \\
.1021\end{array}$ & $\begin{array}{l}.0002 \\
.0003 \\
.0004 \\
.0001 \\
.0002\end{array}$ & $\begin{array}{l}.00011 \\
.00013 \\
.00014 \\
.00015 \\
.00016\end{array}$ \\
\hline $\begin{array}{l}.12 \\
.14 \\
.16 \\
.18 \\
.2\end{array}$ & $\begin{array}{l}.11886 \\
.13681 \\
.15737 \\
.17806 \\
.20071\end{array}$ & $\begin{array}{l}.11884 \\
.13760 \\
.15765 \\
.17792 \\
.20089\end{array}$ & $\begin{array}{l}.11951 \\
.13819 \\
.15703 \\
.17888 \\
.20041\end{array}$ & $\begin{array}{l}.1191 \\
.1375 \\
.1573 \\
.1783 \\
.2007\end{array}$ & $\begin{array}{l}.0004 \\
.0007 \\
.0003 \\
.0006 \\
.0003\end{array}$ & $\begin{array}{l}.00019 \\
.00021 \\
.00024 \\
.00026 \\
.00029\end{array}$ \\
\hline $\begin{array}{l}.25 \\
.5 \\
1 \\
2 \\
3\end{array}$ & $\begin{array}{l}.24702 \\
.49799 \\
1.0037 \\
1.9954 \\
2.9968\end{array}$ & $\begin{array}{l}.24709 \\
.49762 \\
1.0037 \\
1.9972 \\
2.9975\end{array}$ & $\begin{array}{l}.24724 \\
.49795 \\
1.0037 \\
1.9948 \\
2.9993\end{array}$ & $\begin{array}{l}.2471 \\
.4979 \\
1.004 \\
1.996 \\
2.998\end{array}$ & $\begin{array}{l}.0001 \\
.0003 \\
.0000 \\
.0010 \\
.0015\end{array}$ & $\begin{array}{l}.00035 \\
.00065 \\
.0013 \\
.0029 \\
.0047\end{array}$ \\
\hline $\begin{array}{r}5 \\
10 \\
15 \\
20 \\
25\end{array}$ & $\begin{array}{c}\text { 4. } 9841 \\
9.9624 \\
14.991 \\
19.963 \\
24.999\end{array}$ & $\begin{array}{c}4.9923 \\
9.9671 \\
14.988 \\
19.956 \\
25.031\end{array}$ & $\begin{array}{c}4.9927 \\
9.9647 \\
15.001 \\
19.945 \\
24.987\end{array}$ & $\begin{array}{c}4.990 \\
9.965 \\
14.99 \\
19.95 \\
25.01\end{array}$ & $\begin{array}{l}.006 \\
.003 \\
.000 \\
.01 \\
.02\end{array}$ & $\begin{array}{l}.0048 \\
.0053 \\
.0063 \\
.0093 \\
.014\end{array}$ \\
\hline $\begin{array}{l}30 \\
40 \\
50\end{array}$ & $\begin{array}{l}30.080 \\
40.354 \\
52.338\end{array}$ & $\begin{array}{l}30.049 \\
40.281 \\
52.041\end{array}$ & $\begin{array}{l}30.074 \\
40.367 \\
52.336\end{array}$ & $\begin{array}{l}30.07 \\
40.33 \\
52.24\end{array}$ & $\begin{array}{l}.02 \\
.05 \\
.20\end{array}$ & $\begin{array}{l}.015 \\
.02 \\
.06\end{array}$ \\
\hline
\end{tabular}

\section{Appendix}

The analysis (see footnote 4 ) of mismatch errors in the calibration of variable attenuators yielded an equation for the error in terms of the scattering coefficients of two fourpoles corresponding to two settings of the attenuator dial, and the reflection coefficients of the system in which the attenuator was inserted. The measurement of all these quantities may be tedious or difficult, and an approximate method has been developed. One obtains reasonably close limits within which the error lies from a fairly simple experimental procedure.

The complete expression for the mismatch error is

$$
\epsilon=20 \log _{10}\left|\frac{\left(1-S_{11}^{\prime} \Gamma_{G}\right)\left(1-S_{22}^{\prime} \Gamma_{L}\right)-\left(S_{21}^{\prime}\right)^{2} \Gamma_{G} \Gamma_{L}}{\left(1-S_{11} \Gamma_{G}\right)\left(1-S_{22} \Gamma_{L}\right)-\left(S_{21}\right)^{2} \Gamma_{G} \Gamma_{L}}\right|,
$$

where scattering coefficients are denoted by $S_{m n}$, and $\Gamma_{G}, \Gamma_{L}$ represent, respectively, the reflection coefficients of the system "looking towards" the generator and load. Primes are used to designate a setting of the attenuator other than the zero or reference setting.

For small reflections, the following expression is derived from (1a):

$$
\epsilon \approx 20 \log _{10}\left|1-\left(S_{11}^{\prime}-S_{11}\right) \Gamma_{G}-\left(S_{22}^{\prime}-S_{22}\right) \Gamma_{L}+\left[S_{11}^{\prime} S_{22}^{\prime}-S_{11} S_{22}-\left(S_{21}^{\prime}\right)^{2}+S_{21}^{2}\right] \Gamma_{G} \Gamma_{L}\right|
$$

If the attenuator VSWR is small, the products $S^{\prime}{ }_{11} S_{22}^{\prime}$ and $S_{11} S_{22}$ may be neglected. Then (2a) becomes

$$
\epsilon \approx 20 \log _{10}\left|1+\left(S_{11}-S_{11}^{\prime}\right) \Gamma_{G}+\left(S_{22}-S_{22}^{\prime}\right) \Gamma_{L}+\left[S_{21}{ }^{2}-\left(S_{21}^{\prime}\right)^{2}\right] \Gamma_{G} \Gamma_{L}\right|
$$

It is convenient to determine the magnitudes of the individual terms but not their phases, so that the limit of error, allowing random phase variations in $S_{11}$ and $S_{22}$, but none in $S_{21}$ is

$$
\epsilon \approx 20 \log _{10}\left[1+\left|S_{11}^{\prime}-S_{11}\right|\left|\Gamma_{G}\right|+\left|S_{22}^{\prime}-S_{22}\right|\left|\Gamma_{L}\right|+\left(\left|S_{21}\right|^{2}-\left|S_{21}^{\prime}\right|^{2}\right)\left|\Gamma_{G} \Gamma_{L}\right|\right]
$$


The quantity $\left|S_{11}-S_{11}^{\prime}\right|$ is determined as follows. With the attenuator under test connected as shown in figure 5, and set to its zero or reference position, tuner $\mathrm{A}$ is adjusted for a detector null, and tuner $\mathrm{B}$ is then adjusted until the reflection coefficient $\Gamma_{2 i}$ of the equivalent generator at terminal plane 2 vanishes. (This condition may be recognized by means of an auxiliary reflectometer.)

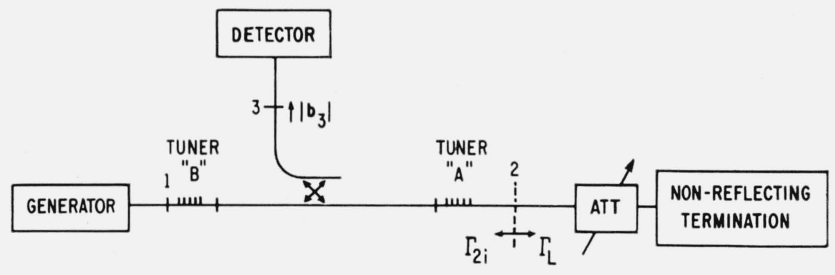

FIGURE 5. Schematic of system for measuring $\left|S_{11}-S_{11}^{\prime}\right|$.

Movement of the attenuator dial to some other setting will then give an observable detector output. Using the theory ${ }^{6}$ of a directional coupler with auxiliary tuners, one can obtain $\left|S_{11}-S_{11}^{\prime}\right|$ in the following way.

The amplitude of the output power from the sidearm of the directional coupler in figure 5 has the magnitude $\left|b_{3}\right|=k\left|\frac{1+K \Gamma_{L}}{1-\Gamma_{2 i} \Gamma_{L}}\right|$, where $k$ is a constant for a given stable generator operating level.

With the attenuator set first on zero, $\Gamma_{L}=S_{11}$. Adjusting tuner A for $\left|b_{3}\right|=0$ changes $K$ so that $K S_{11}=-1$. Adjusting tuner $\mathrm{B}$ for $\Gamma_{2 i}=0$ makes the dependence of $\left|b_{3}\right|$ on $\Gamma_{L}$ take the simple form:

$$
\left|b_{3}\right|=k\left|1-\frac{\Gamma_{L}}{S_{11}}\right|
$$

Suppose the attenuator dial is moved to a new position such that $\Gamma_{L}$ now equals $S_{11}^{\prime}$; then $\left|b_{3}\right|=k\left|1-\frac{S_{11}^{\prime}}{S_{11}}\right|=\frac{k}{\left|S_{11}\right|}\left|S_{11}-S_{11}^{\prime}\right| \cdot \quad$ The factor $k /\left|S_{11}\right|$ is obtained by replacing the attenuator by a waveguide section containing a sliding short. Upon sliding the short, $\left|b_{3}\right|$ goes through small variations so that one may observe $\left|b_{3}\right|_{\max }$ and $\left|b_{3}\right|_{\min }$. It is easily shown that

$$
\frac{k}{\left|S_{11}\right|}=\frac{1}{2}\left(\left|b_{3}\right|_{\max }+\left|b_{3}\right|_{\mathrm{min}}\right) \text {. }
$$

One can assume that $\left|b_{3}\right|_{\max }=\left|b_{3}\right|_{\min }$ and employ a fixed short circuit with negligible error if the VSWR corresponding to $S_{11}$ is less than 1.15. It may be that this error is tolerable for higher VSWR's since it is not important to know $\left|S_{11}-S_{11}^{\prime}\right|$ to great accuracy.

The quantity $\left|S_{22}-S_{22}^{\prime}\right|$ is found in the same way as above with the attenuator turned end for end.

The authors acknowledge the assistance of James E. Gilbert and Morris E. Harvey in making the attenuation measurements and of Wilbur J. Anson and Ronald Ridge in matching the system and making measurements to evaluate the limits of mismatch error. G. E. Schafer read the manuscript and provided information on phase shifts in variable attenuators.

\footnotetext{
${ }^{6}$ R. W. Beatty and D. M. Kerns, Recently developed microwave impedance standards and methods of measurement, IRE Trans. on Instr., I-7, Nos. $3 \& 4,319-321$ (Dec. 1958).
}

Boulder, Colo.

(Paper 64C2-33) 